\title{
Diagnostics and Aging of Electrically Conductive Adhesives
}

P. Mach, M. Škvor, P. Száraz

\section{INTRODUCTION}

Diagnostics of electrically conductive adhesives is very sigrificant for development as well as optimum application of these very promising materials. There are different parameters, which are used for evaluation of ECA. As to electrical properties resistance of adhesive joints is usually measured. Mechanical properties are evaluated by tensile strength or shear strength. With respect to the material of the filler of ECA (silver or copper plated by silver is used very often) migration of silver ions is also investigated.

Analysis of electrical conductivity of ECA is very complicated. Different mechanisms, e.g. phonon-electron interaction, tunneling through barriers among adjacent grains, Schottky emission and others probably influence the total conductivity of the joint. Some of these mechanisms are linear, e.g. phonon-electron interaction, and some nonlinP. Mach, M. Skvor and P. Sxarax are with the Department of Electrotechnology. Facnlty of Electrical Engineering, Czech Tectuical University in Pregue, Technická 2, 16627 Prague 6, Czech Republic. Emaili mach@fetd.crut.cz, skvorml@feld.cvut.cz, xszaraz@feld.cvut.cz ear, e.g. tumeling or Schottky emission. According to the Holm's model [1] of a point contact it can be estimated that for the contact with the resistance of $100 \mathrm{~m} \Omega$ the radius of a contact spot is approximately $100 \mathrm{tm}$. Therefore very high current densities can occur here $\left(10^{4}-10^{6} \mathrm{~A} / \mathrm{mm}^{2}\right)$. Such the current density can change the temperature of the contact spot substantially and cause noise and nonlinearity of this contact, as well.

Thermal aging of the adhesive bonds causes decrease of their volume. Therefore the conductive particles of the filler more squeezed and the quality of contacts among them improves. Therefore resistance of the joints usully decreases during the first period of themal aging.

Distribution of conductive particles inside the adhasive joints is usually investigated using SEM. Electrical methods, which make study of changes of distribution of conductive particles inside adhesive possible are measurement of noise and measurement of nonlinearity of current $\mathrm{Ys}$. voltage characteristic (NAVO).

Basic principle and some results of NAVC measurement are presented in this paper.

\section{BASIC THEORY OF NONLINEARTTY}

The basic theory of nonlinearity was given by J. C. Anderson and V, Rysánek [2] for the thin films but its conclusions can be generalized. This theory describes a relationship anong inhomogenities and other defec1s inside the film, its resistance and nonlinearity of its curtent vs. voltage characteristic. The busic assumption of the theory is given by the Matthiesen's rule

$R_{T}=R_{D}+R$

Where $R_{T}$ is the total film resistance and $R$ is a constant portion of it that arises from normal scattering processes. 
$R_{D}$ due to barriers among the conductive areas reptesents nonlinear scattering processes, which causes nonlinearity of the $C-V$ charaoteristic.

If $i$-th boundary botween two conductive cubic areas represents the potential barrier of $V_{1}$ volts to conduction electrons then, under thermal equilibrium, the current $I_{i}$ across the bartier in any direction will be given by equation (2) where $A$ is a constant depending on the material of the film. It was derived that the net increase in the total current $I_{D}$ across the $N_{1}$ barriers can be described by equation (3) where $V$ is the voltage applied across the barriers. It is assumed that all the barriers are of the same type.

$I_{i}=A \exp \left(\frac{-\ell V_{i}}{k T}\right)$

$I_{D}=2 N_{t} A \exp \left(\frac{-e V_{i}}{k T}\right) \sinh \left(\frac{e V}{k T}\right)$

Expanding of the sinh term ith a series a dependence of the total current $I_{D}$ on the harmonic components of the applied voltage can be found (4). The terms of the series are given by odd powers of the applied voltage only, Therefore the $\mathrm{CrV}$ characteristic is symmetrical and its nonlinearity can be evaluated according to the amplitude of thitc harmonic component.

$I_{D} \approx\left(\frac{e V}{k T}+\frac{e^{3} V^{s}}{3 !(k T)^{3}}+\ldots ..\right)$

The nonlinearity measurement of a current vs. voltage characteristic differs according to the type of nonlinearity monitored. There are two possible ways how to perform this measurement - to measure nonlinearity of the $\mathrm{C}-\mathrm{V}$ characteristic or to measure differential nonlinearity of this characteristic.

When nonlinearity is investigated the component under tost is fed by the sinusoidal current [3] and the third harmonic component of the alternative voltage across the component is measured. The amplitude of the current is changed and the voltage of the third harmonics is measured on every level of the current. Usually the evaluation of the results is carried out by the following way: a diagram of $U_{3}$ (the voltage of third harmonics across the component in $\mu \mathrm{V}$ ) ys. $\mathrm{U}_{1}$ (the voltage of the basic harmonics across the component in $\mathrm{V}$ ) in $\log \log$ coordinates is drawn. The measured values are fitted by a straight line. When nonlinearity of the current vs. voltage characteristic is a cubical parabola then $\operatorname{tg} \alpha$, where $\alpha$ is defined as $\Delta U_{3}(\mu V) / \Delta U_{1}$ (V), is equal to 3 . When nonlinearity is symmetrical but it is not the pure cubical parabola the value of $\mathrm{tg}$ o differs from number 3. According to the theory of nonlinearity cubical nonlinearity will dominate and therefore the value of tg $\alpha$ should be near to 3 . When a difference between the value of $\operatorname{tg} \alpha$ and 3 is higher it is necessary to be very careful in conclusions made on the base of the nonlineatity measurements because such the case beggars theory and therefore a deeper analysis is necessary. Nonlinearity is usually expressed as a "Third harmonics index" THI which is calculated according to the equation:

$$
T H I=20, \log \frac{U_{3}}{U_{1}^{n}}
$$

monics index, $\mathrm{U}_{3}(\mu \mathrm{V})$... the voltage of the third harmonic component, $U_{1}(V)$... the voltage of the basic harmonics which is generated across the component by its feeding with the sinusoidal current, $n=3$ when nonlinearity of the C-V characteristics can be described by a cubical parabola.

\section{Preparation of Samples}

The samples of adhesive joints were prepared on Cu clad FR4 laminate. Ten resistors with "zoro value" (their factual value was $5 \mathrm{~m} \Omega$ ) recommended for assembly using ECA were assembled on one substrate therefore twenty adhesive joints were for disposal on one substrate. Four types of adhesives were tested: Amepox, Alpha Metals 171, Alpha Metals 181, and Epoxy Technology E4110. Conductive tracks were designed to allow the measurements of one 
joint or more joints connected seriously. Both the resistance and NAVC measurements were carried out in the four-point arrangement.

Some samples were thermally aged at the temperature of $160^{\circ} \mathrm{C}$ for 6 hours. Aging was performed in a precision ovea. We realized this aging to recognize sensitivity of NAVC measurement to changes caused by aging of adhesives.

\section{EXPERTMENTAL RESULTS AND DISCUSSION}

Difference between the measurement of the resistance and THI of the samples is shown in Fig. 1. It was found that nonlinearity is substantially more sensitive to changes caused in adhesive joints by thermal aging. Therefore it seems that measurement of THI should complete the measurement of resistance, which is usually performed.

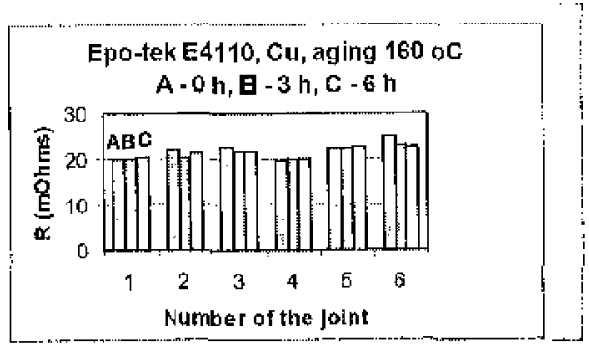

Fig. 1. Resistance of the adhesive joints prepared of adlesive E4 110 on copper, Joints were aged at the temperature of $160^{\circ} \mathrm{C}$

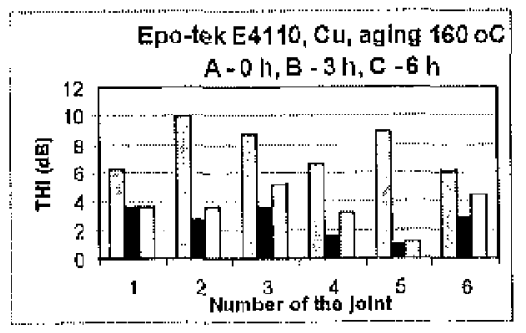

Fig 2. Nonlinearity of the adhesive joints prepared of adhesive $E 4 I 10$ on copper. Joints were aged at the temperalure of $160^{\circ} \mathrm{C}$
Very interesting is also comparison of THI at the first period of aging (after 3 hours) and after the second period of aging (after 6 hours). It is possible to observe improvement of quality of the joints, which is caused by decrease of their volume after the first period. It calses better contact among conductive particles and decrease of nonlinearity. Further aging creates probably formation of oxide layers on metal particles and therefore quality of the joints decreases.

Another example of the use of the measurement of non linearity is shown in Fig. 3 and in Fig. 4.

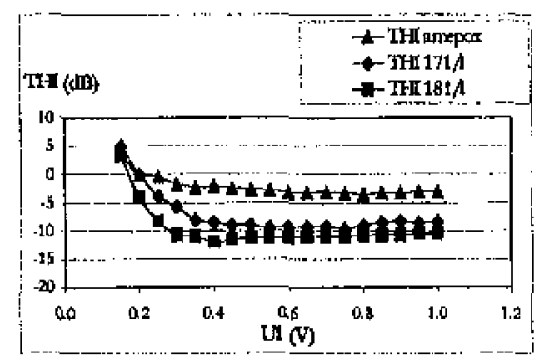

Fig. 3. Nonlinearity of the adhesive joints prepared of adhesives Amepox, Alpha Metals 171 and Alpha Metals 181 on copper Joints were not aged

Three types of ECA were measured and changes of THI were compared reciprocally before and after thermal aging at the temperature of $200^{\circ} \mathrm{C}$. It is possible to observe dependence of THI on the amplitude of the basic harmonic component. The nonlinerarily of the characteristics THI vs. $\mathrm{U}_{1}$ decreased after thermal aging of adhesives of the types 171 and 181 . The change of these characteristics toward to a straight line is joined with a strong increase of the value of 'THI. This fact can be caused by creating of stabile tunneling junctions with defined nonlinearity. Cubical current Ys, voltage characteristic is joined with elastic tunneling. It is possible that elementary tumeling micro-junctions among conductive particles created by aging inside adhe- 
sive dominate and mechanisms of other types which cause nonlinearity do not come into play.

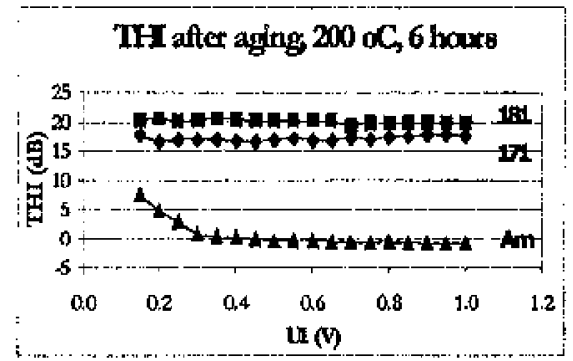

Fig. 4 Nonlinearity of the adhesive joints prepared of adhesives Amepox, Alpha Metals 171 and Alpha Metals 181 on copper. Joints were not aged

\section{CONCLUSIONS}

The use of nonlinearity measurement is not experimentally exacting. Therefore it could be spread as a method which will complete a group of diagnostic methods which are used for analysis of ECA presently. Measurement of resistance is not sufficient for deeper analysis of ECA because resistance is an integral magnitude, which is given as the result of mutual incidence of different mechanisms. Nonlinearity is also influenced by different mechanisms of conductivity. However, it seems that infuence of these mechanisms on resistance and nonlinearity is different, Therefore combination of these parameters can be very useful.

Measurement of noise is a method, which could also contribute to analysis of conductivity mechanisms of ECA and to analysis of changes, which occur in these materials after their aging. According to the theory [4] the causes of noise and nonlinearity are the same. Therefore both these parameters should be influenced by the same mechanisms, From this point of view nonlinearity measurement can substitute noise measurement and vice versa. On the other hand the measurement of $1 / f$ noise can be used for analysis at low frequency (some $\mathrm{Hz}$ ) and nonlinearity measurement at higher frequency (some $\mathrm{kHz}$ or $\mathrm{MHz}$, respectively).
The adhesive bonds were prepared of four types of ECA. Nonlinearity of adhesives of the types 171 and 172 increased after thermal aging substantially. The most probable reason of these changes is formation of very thin insulating layers on surface of conductive particles. It was also found that nonlinearity of this newly created structure does not depend on amplitude of the basic harmonics. Adhesive Amepox did not change nonlinearity after thermal aging.

Measurement of nonlinearity of current vs. voltage characteristic of adhesive bonds was investigated. It was found that this measurement could be very useful for analysis of mechanism of changes, which occurs in ECA during aging. It was also found that nonlinearity is more sensitive to these changes than resistance of the bonds.

\section{ACKNOWLEDGMENT}

This project was encouraged by the grant No, F $759 / 99$ of the Ministry of Education of the Czech Republic

\section{REFERENCES}

[1] R. Holm and E. Holm, Electrtc Contacts Handbook, Springer Verlag, Berlin, Germany, 1958

[2] J. C. Anderson and V. Rysanek, „Prediction of the Stability of Thin-film Resistors, "The Rudis and Electronic Engineer, Vol. 39, No. 6, pp. 321-327, June 1970

[3] V. Rysanek, C. Corsi, A. D'Amico, „Nonlinearity neasurements using alternative current", Electrocomponent Sclence and Techtholagy. Vol. 5, pp, 614-621, 1978

[4] G. P. Zhigalskij, "Noise of the type $1 / f$ and nonlinear effects in thin metallic films (in Russian)", Uspech fizi Geskich nouk, Vol. 167 . No. 5, pp, 624-648, 1997

[3] Ogiube, Ch. N. ef al., Conduction Mechanisms in Ajisotropic Conducting Adhesive Assembly, IEEL 7 Tams. on Comp, Pack. and Manuf. Technology, Part A, Vol. 21, No. 2, pp. 235-242, 1998

[6] Liu, J. et al., "Sufface characteristics, reliability and failure mechanisms of tin, copper and gold metalizations", Proc. Adhesive Blectron. 96, Stockhalm, Sweden, pp. 141-153, 1996

[7] $\mathrm{Li}_{1} \mathrm{~L}$ ef al, "Electrical, Structurat and Processing Properties of Electrically Conductive Adtosives, LEEE Trans, Comp, Hybrids, Mawufact. Technol., Vol. 16, jp. 205-214, 1993 